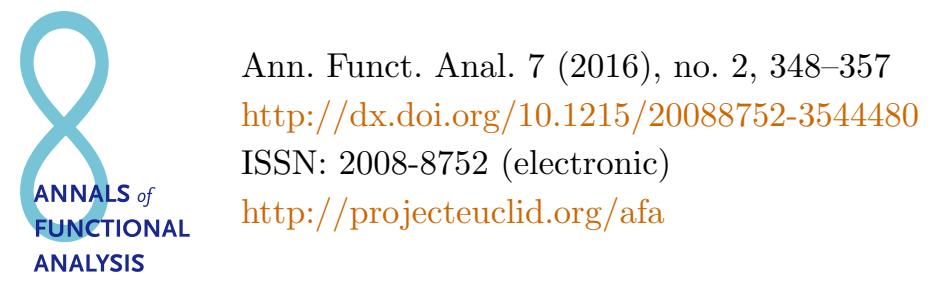

\title{
SOME MATRIX INEQUALITIES FOR WEIGHTED POWER MEAN
}

\author{
MARYAM KHOSRAVI
}

Communicated by Y. Seo

ABSTRACT. In this paper, we prove that, for any positive definite matrices $A, B$, and real numbers $\nu, \mu, p$ with $-1 \leq p<1$ and $0<\nu \leq \mu<1$, we have

$$
\frac{\nu}{\mu}\left(A \nabla_{\mu} B-A \sharp_{p, \mu} B\right) \leq A \nabla_{\nu} B-A \sharp_{p, \nu} B \leq \frac{1-\nu}{1-\mu}\left(A \nabla_{\mu} B-A \sharp_{p, \mu} B\right),
$$

where $\nabla_{\nu}$ and $\sharp_{p, \nu}$ stand for weighted arithmetic and power mean, respectively. In the special cases when $p=0,1$, this inequality can be considered as a generalization of harmonic-arithmetic and geometric-arithmetic means inequalities and their reverses.

Applying this inequality, some inequalities for the Heinz mean and determinant inequalities related to weighted power means are obtained.

\section{INTRODUCTION}

Let $\mathbb{B}(H)$ be the algebra of bounded linear operators on a Hilbert space $H$, and let $\mathbb{B}(H)^{+}$stand for the strictly positive operators. A binary operation $(A, B) \mapsto$ $A \sigma B$, from $\mathbb{B}^{+}(H) \times \mathbb{B}^{+}(H)$ to $\mathbb{B}^{+}(H)$, is called a connection if the following conditions are satisfied:

- $A \leq C$ and $B \leq D$ imply $A \sigma B \leq C \sigma D$,

- $A_{n} \downarrow A$ and $B_{n} \downarrow B$ imply $A_{n} \sigma B_{n} \downarrow A \sigma B$ in the strong operator topology,

- $T^{*}(A \sigma B) T \leq\left(T^{*} A T\right) \sigma\left(T^{*} B T\right)$ for every operator $T$.

Copyright 2016 by the Tusi Mathematical Research Group.

Received Jun. 29, 2015; Accepted Oct. 18, 2015.

2010 Mathematics Subject Classification. Primary 15A45; Secondary 47A64, 26 E60.

Keywords. weighted arithmetic mean, weighted power mean, positive definite matrices, matrix inequality. 


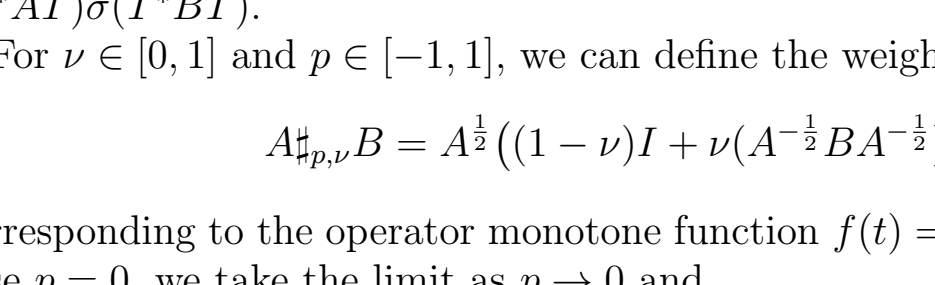

\title{
SOME MATRIX INEQUALITIES FOR WEIGHTED POWER MEAN
}

\author{
MARYAM KHOSRAVI
}

Communicated by Y. Seo

ABSTRACT. In this paper, we prove that, for any positive definite matrices $A, B$, and real numbers $\nu, \mu, p$ with $-1 \leq p<1$ and $0<\nu \leq \mu<1$, we have

$$
\frac{\nu}{\mu}\left(A \nabla_{\mu} B-A \sharp_{p, \mu} B\right) \leq A \nabla_{\nu} B-A \sharp_{p, \nu} B \leq \frac{1-\nu}{1-\mu}\left(A \nabla_{\mu} B-A \sharp_{p, \mu} B\right),
$$

where $\nabla_{\nu}$ and $\sharp_{p, \nu}$ stand for weighted arithmetic and power mean, respectively. In the special cases when $p=0,1$, this inequality can be considered as a generalization of harmonic-arithmetic and geometric-arithmetic means inequalities and their reverses.

Applying this inequality, some inequalities for the Heinz mean and determinant inequalities related to weighted power means are obtained.

\section{INTRODUCTION}

Let $\mathbb{B}(H)$ be the algebra of bounded linear operators on a Hilbert space $H$, and let $\mathbb{B}(H)^{+}$stand for the strictly positive operators. A binary operation $(A, B) \mapsto$ $A \sigma B$, from $\mathbb{B}^{+}(H) \times \mathbb{B}^{+}(H)$ to $\mathbb{B}^{+}(H)$, is called a connection if the following conditions are satisfied:

- $A \leq C$ and $B \leq D$ imply $A \sigma B \leq C \sigma D$,

- $A_{n} \downarrow A$ and $B_{n} \downarrow B$ imply $A_{n} \sigma B_{n} \downarrow A \sigma B$ in the strong operator topology,

- $T^{*}(A \sigma B) T \leq\left(T^{*} A T\right) \sigma\left(T^{*} B T\right)$ for every operator $T$.

Copyright 2016 by the Tusi Mathematical Research Group.

Received Jun. 29, 2015; Accepted Oct. 18, 2015.

2010 Mathematics Subject Classification. Primary 15A45; Secondary 47A64, 26 E60.

Keywords. weighted arithmetic mean, weighted power mean, positive definite matrices, matrix inequality. 


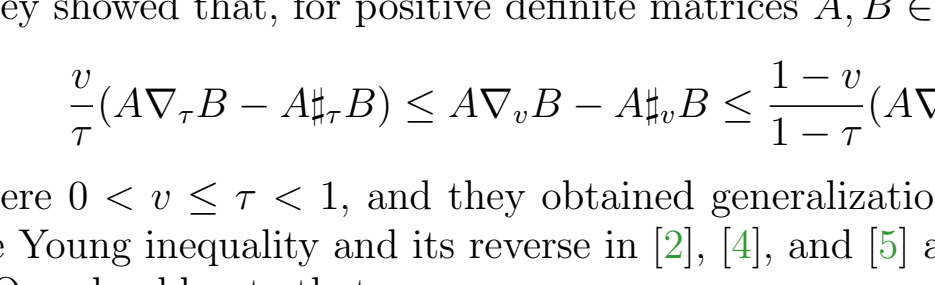

\title{
SOME MATRIX INEQUALITIES FOR WEIGHTED POWER MEAN
}

\author{
MARYAM KHOSRAVI
}

Communicated by Y. Seo

ABSTRACT. In this paper, we prove that, for any positive definite matrices $A, B$, and real numbers $\nu, \mu, p$ with $-1 \leq p<1$ and $0<\nu \leq \mu<1$, we have

$$
\frac{\nu}{\mu}\left(A \nabla_{\mu} B-A \sharp_{p, \mu} B\right) \leq A \nabla_{\nu} B-A \sharp_{p, \nu} B \leq \frac{1-\nu}{1-\mu}\left(A \nabla_{\mu} B-A \sharp_{p, \mu} B\right),
$$

where $\nabla_{\nu}$ and $\sharp_{p, \nu}$ stand for weighted arithmetic and power mean, respectively. In the special cases when $p=0,1$, this inequality can be considered as a generalization of harmonic-arithmetic and geometric-arithmetic means inequalities and their reverses.

Applying this inequality, some inequalities for the Heinz mean and determinant inequalities related to weighted power means are obtained.

\section{INTRODUCTION}

Let $\mathbb{B}(H)$ be the algebra of bounded linear operators on a Hilbert space $H$, and let $\mathbb{B}(H)^{+}$stand for the strictly positive operators. A binary operation $(A, B) \mapsto$ $A \sigma B$, from $\mathbb{B}^{+}(H) \times \mathbb{B}^{+}(H)$ to $\mathbb{B}^{+}(H)$, is called a connection if the following conditions are satisfied:

- $A \leq C$ and $B \leq D$ imply $A \sigma B \leq C \sigma D$,

- $A_{n} \downarrow A$ and $B_{n} \downarrow B$ imply $A_{n} \sigma B_{n} \downarrow A \sigma B$ in the strong operator topology,

- $T^{*}(A \sigma B) T \leq\left(T^{*} A T\right) \sigma\left(T^{*} B T\right)$ for every operator $T$.

Copyright 2016 by the Tusi Mathematical Research Group.

Received Jun. 29, 2015; Accepted Oct. 18, 2015.

2010 Mathematics Subject Classification. Primary 15A45; Secondary 47A64, 26 E60.

Keywords. weighted arithmetic mean, weighted power mean, positive definite matrices, matrix inequality. 


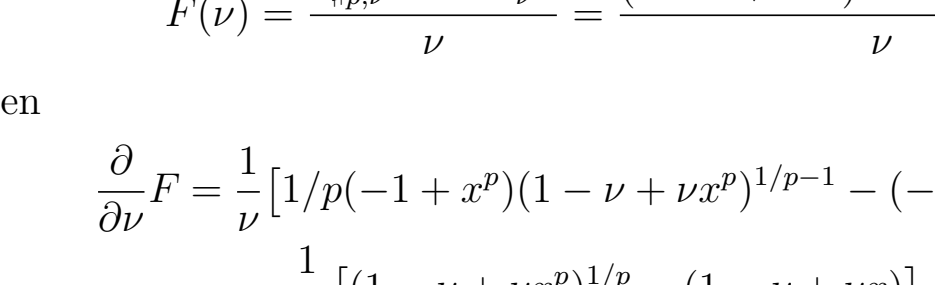

\title{
SOME MATRIX INEQUALITIES FOR WEIGHTED POWER MEAN
}

\author{
MARYAM KHOSRAVI
}

Communicated by Y. Seo

ABSTRACT. In this paper, we prove that, for any positive definite matrices $A, B$, and real numbers $\nu, \mu, p$ with $-1 \leq p<1$ and $0<\nu \leq \mu<1$, we have

$$
\frac{\nu}{\mu}\left(A \nabla_{\mu} B-A \sharp_{p, \mu} B\right) \leq A \nabla_{\nu} B-A \sharp_{p, \nu} B \leq \frac{1-\nu}{1-\mu}\left(A \nabla_{\mu} B-A \sharp_{p, \mu} B\right),
$$

where $\nabla_{\nu}$ and $\sharp_{p, \nu}$ stand for weighted arithmetic and power mean, respectively. In the special cases when $p=0,1$, this inequality can be considered as a generalization of harmonic-arithmetic and geometric-arithmetic means inequalities and their reverses.

Applying this inequality, some inequalities for the Heinz mean and determinant inequalities related to weighted power means are obtained.

\section{INTRODUCTION}

Let $\mathbb{B}(H)$ be the algebra of bounded linear operators on a Hilbert space $H$, and let $\mathbb{B}(H)^{+}$stand for the strictly positive operators. A binary operation $(A, B) \mapsto$ $A \sigma B$, from $\mathbb{B}^{+}(H) \times \mathbb{B}^{+}(H)$ to $\mathbb{B}^{+}(H)$, is called a connection if the following conditions are satisfied:

- $A \leq C$ and $B \leq D$ imply $A \sigma B \leq C \sigma D$,

- $A_{n} \downarrow A$ and $B_{n} \downarrow B$ imply $A_{n} \sigma B_{n} \downarrow A \sigma B$ in the strong operator topology,

- $T^{*}(A \sigma B) T \leq\left(T^{*} A T\right) \sigma\left(T^{*} B T\right)$ for every operator $T$.

Copyright 2016 by the Tusi Mathematical Research Group.

Received Jun. 29, 2015; Accepted Oct. 18, 2015.

2010 Mathematics Subject Classification. Primary 15A45; Secondary 47A64, 26 E60.

Keywords. weighted arithmetic mean, weighted power mean, positive definite matrices, matrix inequality. 


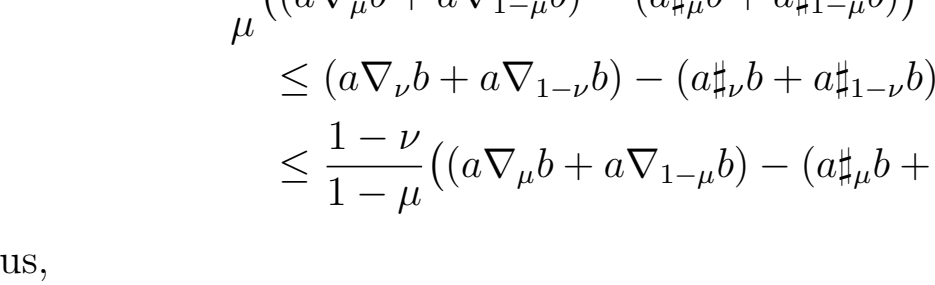

\title{
SOME MATRIX INEQUALITIES FOR WEIGHTED POWER MEAN
}

\author{
MARYAM KHOSRAVI
}

Communicated by Y. Seo

ABSTRACT. In this paper, we prove that, for any positive definite matrices $A, B$, and real numbers $\nu, \mu, p$ with $-1 \leq p<1$ and $0<\nu \leq \mu<1$, we have

$$
\frac{\nu}{\mu}\left(A \nabla_{\mu} B-A \sharp_{p, \mu} B\right) \leq A \nabla_{\nu} B-A \sharp_{p, \nu} B \leq \frac{1-\nu}{1-\mu}\left(A \nabla_{\mu} B-A \sharp_{p, \mu} B\right),
$$

where $\nabla_{\nu}$ and $\sharp_{p, \nu}$ stand for weighted arithmetic and power mean, respectively. In the special cases when $p=0,1$, this inequality can be considered as a generalization of harmonic-arithmetic and geometric-arithmetic means inequalities and their reverses.

Applying this inequality, some inequalities for the Heinz mean and determinant inequalities related to weighted power means are obtained.

\section{INTRODUCTION}

Let $\mathbb{B}(H)$ be the algebra of bounded linear operators on a Hilbert space $H$, and let $\mathbb{B}(H)^{+}$stand for the strictly positive operators. A binary operation $(A, B) \mapsto$ $A \sigma B$, from $\mathbb{B}^{+}(H) \times \mathbb{B}^{+}(H)$ to $\mathbb{B}^{+}(H)$, is called a connection if the following conditions are satisfied:

- $A \leq C$ and $B \leq D$ imply $A \sigma B \leq C \sigma D$,

- $A_{n} \downarrow A$ and $B_{n} \downarrow B$ imply $A_{n} \sigma B_{n} \downarrow A \sigma B$ in the strong operator topology,

- $T^{*}(A \sigma B) T \leq\left(T^{*} A T\right) \sigma\left(T^{*} B T\right)$ for every operator $T$.

Copyright 2016 by the Tusi Mathematical Research Group.

Received Jun. 29, 2015; Accepted Oct. 18, 2015.

2010 Mathematics Subject Classification. Primary 15A45; Secondary 47A64, 26 E60.

Keywords. weighted arithmetic mean, weighted power mean, positive definite matrices, matrix inequality. 


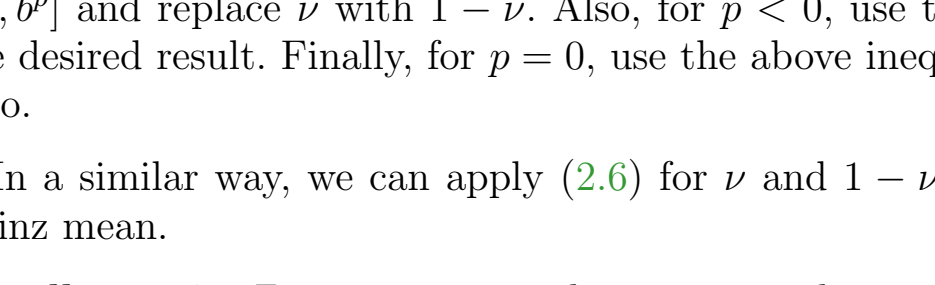

\title{
SOME MATRIX INEQUALITIES FOR WEIGHTED POWER MEAN
}

\author{
MARYAM KHOSRAVI
}

Communicated by Y. Seo

ABSTRACT. In this paper, we prove that, for any positive definite matrices $A, B$, and real numbers $\nu, \mu, p$ with $-1 \leq p<1$ and $0<\nu \leq \mu<1$, we have

$$
\frac{\nu}{\mu}\left(A \nabla_{\mu} B-A \sharp_{p, \mu} B\right) \leq A \nabla_{\nu} B-A \sharp_{p, \nu} B \leq \frac{1-\nu}{1-\mu}\left(A \nabla_{\mu} B-A \sharp_{p, \mu} B\right),
$$

where $\nabla_{\nu}$ and $\sharp_{p, \nu}$ stand for weighted arithmetic and power mean, respectively. In the special cases when $p=0,1$, this inequality can be considered as a generalization of harmonic-arithmetic and geometric-arithmetic means inequalities and their reverses.

Applying this inequality, some inequalities for the Heinz mean and determinant inequalities related to weighted power means are obtained.

\section{INTRODUCTION}

Let $\mathbb{B}(H)$ be the algebra of bounded linear operators on a Hilbert space $H$, and let $\mathbb{B}(H)^{+}$stand for the strictly positive operators. A binary operation $(A, B) \mapsto$ $A \sigma B$, from $\mathbb{B}^{+}(H) \times \mathbb{B}^{+}(H)$ to $\mathbb{B}^{+}(H)$, is called a connection if the following conditions are satisfied:

- $A \leq C$ and $B \leq D$ imply $A \sigma B \leq C \sigma D$,

- $A_{n} \downarrow A$ and $B_{n} \downarrow B$ imply $A_{n} \sigma B_{n} \downarrow A \sigma B$ in the strong operator topology,

- $T^{*}(A \sigma B) T \leq\left(T^{*} A T\right) \sigma\left(T^{*} B T\right)$ for every operator $T$.

Copyright 2016 by the Tusi Mathematical Research Group.

Received Jun. 29, 2015; Accepted Oct. 18, 2015.

2010 Mathematics Subject Classification. Primary 15A45; Secondary 47A64, 26 E60.

Keywords. weighted arithmetic mean, weighted power mean, positive definite matrices, matrix inequality. 


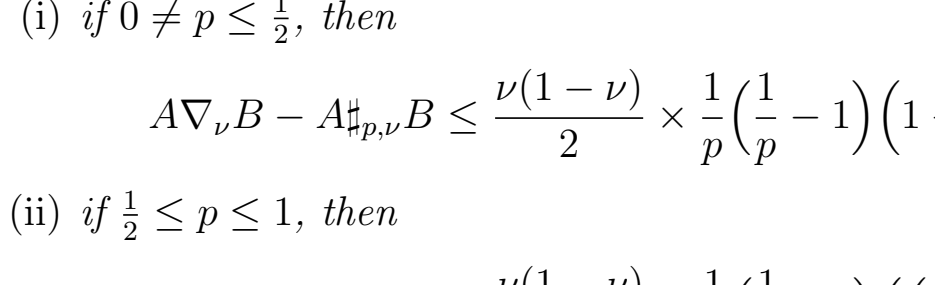

\title{
SOME MATRIX INEQUALITIES FOR WEIGHTED POWER MEAN
}

\author{
MARYAM KHOSRAVI
}

Communicated by Y. Seo

ABSTRACT. In this paper, we prove that, for any positive definite matrices $A, B$, and real numbers $\nu, \mu, p$ with $-1 \leq p<1$ and $0<\nu \leq \mu<1$, we have

$$
\frac{\nu}{\mu}\left(A \nabla_{\mu} B-A \sharp_{p, \mu} B\right) \leq A \nabla_{\nu} B-A \sharp_{p, \nu} B \leq \frac{1-\nu}{1-\mu}\left(A \nabla_{\mu} B-A \sharp_{p, \mu} B\right),
$$

where $\nabla_{\nu}$ and $\sharp_{p, \nu}$ stand for weighted arithmetic and power mean, respectively. In the special cases when $p=0,1$, this inequality can be considered as a generalization of harmonic-arithmetic and geometric-arithmetic means inequalities and their reverses.

Applying this inequality, some inequalities for the Heinz mean and determinant inequalities related to weighted power means are obtained.

\section{INTRODUCTION}

Let $\mathbb{B}(H)$ be the algebra of bounded linear operators on a Hilbert space $H$, and let $\mathbb{B}(H)^{+}$stand for the strictly positive operators. A binary operation $(A, B) \mapsto$ $A \sigma B$, from $\mathbb{B}^{+}(H) \times \mathbb{B}^{+}(H)$ to $\mathbb{B}^{+}(H)$, is called a connection if the following conditions are satisfied:

- $A \leq C$ and $B \leq D$ imply $A \sigma B \leq C \sigma D$,

- $A_{n} \downarrow A$ and $B_{n} \downarrow B$ imply $A_{n} \sigma B_{n} \downarrow A \sigma B$ in the strong operator topology,

- $T^{*}(A \sigma B) T \leq\left(T^{*} A T\right) \sigma\left(T^{*} B T\right)$ for every operator $T$.

Copyright 2016 by the Tusi Mathematical Research Group.

Received Jun. 29, 2015; Accepted Oct. 18, 2015.

2010 Mathematics Subject Classification. Primary 15A45; Secondary 47A64, 26 E60.

Keywords. weighted arithmetic mean, weighted power mean, positive definite matrices, matrix inequality. 


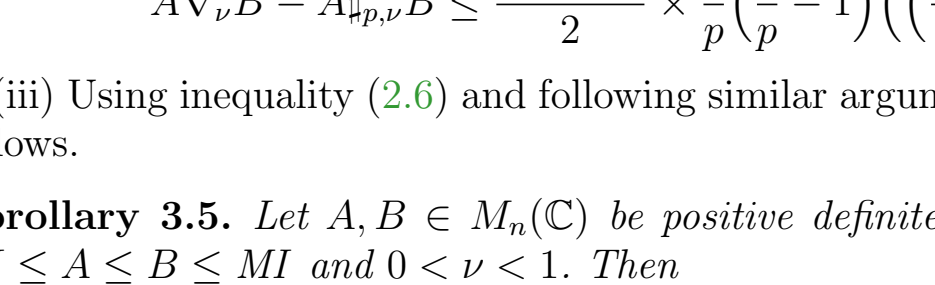

\title{
SOME MATRIX INEQUALITIES FOR WEIGHTED POWER MEAN
}

\author{
MARYAM KHOSRAVI
}

Communicated by Y. Seo

ABSTRACT. In this paper, we prove that, for any positive definite matrices $A, B$, and real numbers $\nu, \mu, p$ with $-1 \leq p<1$ and $0<\nu \leq \mu<1$, we have

$$
\frac{\nu}{\mu}\left(A \nabla_{\mu} B-A \sharp_{p, \mu} B\right) \leq A \nabla_{\nu} B-A \sharp_{p, \nu} B \leq \frac{1-\nu}{1-\mu}\left(A \nabla_{\mu} B-A \sharp_{p, \mu} B\right),
$$

where $\nabla_{\nu}$ and $\sharp_{p, \nu}$ stand for weighted arithmetic and power mean, respectively. In the special cases when $p=0,1$, this inequality can be considered as a generalization of harmonic-arithmetic and geometric-arithmetic means inequalities and their reverses.

Applying this inequality, some inequalities for the Heinz mean and determinant inequalities related to weighted power means are obtained.

\section{INTRODUCTION}

Let $\mathbb{B}(H)$ be the algebra of bounded linear operators on a Hilbert space $H$, and let $\mathbb{B}(H)^{+}$stand for the strictly positive operators. A binary operation $(A, B) \mapsto$ $A \sigma B$, from $\mathbb{B}^{+}(H) \times \mathbb{B}^{+}(H)$ to $\mathbb{B}^{+}(H)$, is called a connection if the following conditions are satisfied:

- $A \leq C$ and $B \leq D$ imply $A \sigma B \leq C \sigma D$,

- $A_{n} \downarrow A$ and $B_{n} \downarrow B$ imply $A_{n} \sigma B_{n} \downarrow A \sigma B$ in the strong operator topology,

- $T^{*}(A \sigma B) T \leq\left(T^{*} A T\right) \sigma\left(T^{*} B T\right)$ for every operator $T$.

Copyright 2016 by the Tusi Mathematical Research Group.

Received Jun. 29, 2015; Accepted Oct. 18, 2015.

2010 Mathematics Subject Classification. Primary 15A45; Secondary 47A64, 26 E60.

Keywords. weighted arithmetic mean, weighted power mean, positive definite matrices, matrix inequality. 


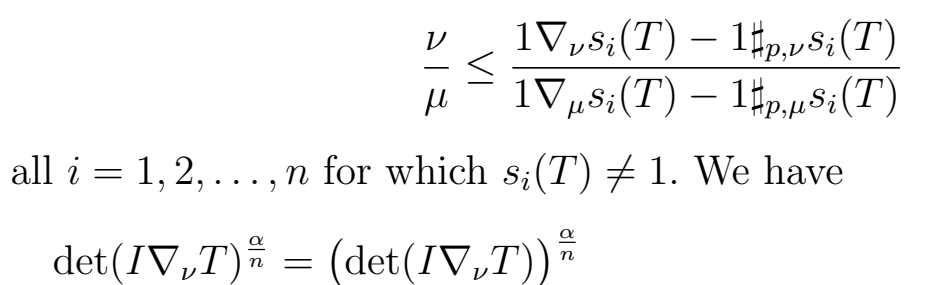

\title{
SOME MATRIX INEQUALITIES FOR WEIGHTED POWER MEAN
}

\author{
MARYAM KHOSRAVI
}

Communicated by Y. Seo

ABSTRACT. In this paper, we prove that, for any positive definite matrices $A, B$, and real numbers $\nu, \mu, p$ with $-1 \leq p<1$ and $0<\nu \leq \mu<1$, we have

$$
\frac{\nu}{\mu}\left(A \nabla_{\mu} B-A \sharp_{p, \mu} B\right) \leq A \nabla_{\nu} B-A \sharp_{p, \nu} B \leq \frac{1-\nu}{1-\mu}\left(A \nabla_{\mu} B-A \sharp_{p, \mu} B\right),
$$

where $\nabla_{\nu}$ and $\sharp_{p, \nu}$ stand for weighted arithmetic and power mean, respectively. In the special cases when $p=0,1$, this inequality can be considered as a generalization of harmonic-arithmetic and geometric-arithmetic means inequalities and their reverses.

Applying this inequality, some inequalities for the Heinz mean and determinant inequalities related to weighted power means are obtained.

\section{INTRODUCTION}

Let $\mathbb{B}(H)$ be the algebra of bounded linear operators on a Hilbert space $H$, and let $\mathbb{B}(H)^{+}$stand for the strictly positive operators. A binary operation $(A, B) \mapsto$ $A \sigma B$, from $\mathbb{B}^{+}(H) \times \mathbb{B}^{+}(H)$ to $\mathbb{B}^{+}(H)$, is called a connection if the following conditions are satisfied:

- $A \leq C$ and $B \leq D$ imply $A \sigma B \leq C \sigma D$,

- $A_{n} \downarrow A$ and $B_{n} \downarrow B$ imply $A_{n} \sigma B_{n} \downarrow A \sigma B$ in the strong operator topology,

- $T^{*}(A \sigma B) T \leq\left(T^{*} A T\right) \sigma\left(T^{*} B T\right)$ for every operator $T$.

Copyright 2016 by the Tusi Mathematical Research Group.

Received Jun. 29, 2015; Accepted Oct. 18, 2015.

2010 Mathematics Subject Classification. Primary 15A45; Secondary 47A64, 26 E60.

Keywords. weighted arithmetic mean, weighted power mean, positive definite matrices, matrix inequality. 


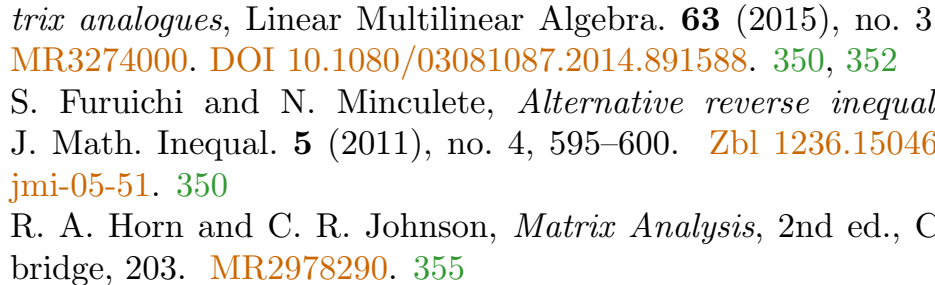

\title{
SOME MATRIX INEQUALITIES FOR WEIGHTED POWER MEAN
}

\author{
MARYAM KHOSRAVI
}

Communicated by Y. Seo

ABSTRACT. In this paper, we prove that, for any positive definite matrices $A, B$, and real numbers $\nu, \mu, p$ with $-1 \leq p<1$ and $0<\nu \leq \mu<1$, we have

$$
\frac{\nu}{\mu}\left(A \nabla_{\mu} B-A \sharp_{p, \mu} B\right) \leq A \nabla_{\nu} B-A \sharp_{p, \nu} B \leq \frac{1-\nu}{1-\mu}\left(A \nabla_{\mu} B-A \sharp_{p, \mu} B\right),
$$

where $\nabla_{\nu}$ and $\sharp_{p, \nu}$ stand for weighted arithmetic and power mean, respectively. In the special cases when $p=0,1$, this inequality can be considered as a generalization of harmonic-arithmetic and geometric-arithmetic means inequalities and their reverses.

Applying this inequality, some inequalities for the Heinz mean and determinant inequalities related to weighted power means are obtained.

\section{INTRODUCTION}

Let $\mathbb{B}(H)$ be the algebra of bounded linear operators on a Hilbert space $H$, and let $\mathbb{B}(H)^{+}$stand for the strictly positive operators. A binary operation $(A, B) \mapsto$ $A \sigma B$, from $\mathbb{B}^{+}(H) \times \mathbb{B}^{+}(H)$ to $\mathbb{B}^{+}(H)$, is called a connection if the following conditions are satisfied:

- $A \leq C$ and $B \leq D$ imply $A \sigma B \leq C \sigma D$,

- $A_{n} \downarrow A$ and $B_{n} \downarrow B$ imply $A_{n} \sigma B_{n} \downarrow A \sigma B$ in the strong operator topology,

- $T^{*}(A \sigma B) T \leq\left(T^{*} A T\right) \sigma\left(T^{*} B T\right)$ for every operator $T$.

Copyright 2016 by the Tusi Mathematical Research Group.

Received Jun. 29, 2015; Accepted Oct. 18, 2015.

2010 Mathematics Subject Classification. Primary 15A45; Secondary 47A64, 26 E60.

Keywords. weighted arithmetic mean, weighted power mean, positive definite matrices, matrix inequality. 